\title{
International volunteerism and urethral stricture disease: a review
}

\author{
Jason K. Frankel ${ }^{1}$, Gregory P. Murphy ${ }^{2}$ \\ ${ }^{1}$ University of Connecticut Health Center, Farmington, CT, USA; ${ }^{2}$ Department of Urology, Washington University, St. Louis, MO, USA \\ Contributions: (I) Conception and design: All authors; (II) Administrative support: All authors; (III) Provision of study materials or patients: All \\ authors; (IV) Collection and assembly of data: All authors; (V) Data analysis and interpretation: All authors; (VI) Manuscript writing: All authors; (VII) \\ Final approval of manuscript: All authors. \\ Correspondence to: Gregory P. Murphy, MD. Assistant Professor of Urologic Surgery, Washington University School of Medicine, 4960 Children's \\ Place, Suite 216, 2 Wohl Hospital, St. Louis, MO 63110, USA. Email: murphyg@wustl.edu.
}

\begin{abstract}
Global health is an ever-expanding area of interest for many healthcare workers around the world. In recent years, it has become apparent that much of the global disease burden is surgical. Urologic disease is no exception-many international organizations send volunteers around the world to support Urologic services in countries that lack capacity and resources. Urethral stricture represents a unique opportunity for specialized surgical management that vastly improves long term morbidity. Here we review the prevalence, etiology, and management of urethral stricture from a global perspective while highlighting impact of international urologic volunteer efforts.
\end{abstract}

Keywords: Urethral stricture; global health; international volunteerism

Submitted Dec 08, 2017. Accepted for publication Feb 08, 2018.

doi: 10.21037/tau.2018.02.04

View this article at: http://dx.doi.org/10.21037/tau.2018.02.04

\section{Introduction}

There have been vast advances in global health over the past 25 years. Many of these advances have been the result of efforts sparked by the Millennium Development Goals (MDGs). These goals were elucidated by the Millennium Summit in 2000 and focused on varying topics including eliminating extreme poverty, greater access to education, gender equality, and improvement in global health care (1). Within the realm of Global Health, the focus was placed on HIV/AIDS, tuberculosis, malaria, and other curable infectious disease. Curable infectious disease mostly refers to neglected tropical diseases (NTD), which include age old maladies such as guinea worm, schistosomiasis, and leprosy (2). The MDGs were first established with the deadline of 2015 , at which point significant progress was to be expected. This was indeed the case, and great strides have been made concerning the treatment of infectious disease (3). Global surgical disease, however, has been mostly neglected over this time. We now know that the burden of global surgical disease vastly outnumbers the burden of HIV,
Tuberculosis, and Malaria combined. In 2010, an estimated 16.9 million deaths around the world could be attributed to conditions needing surgical care (4). Deaths from HIV/ AIDS, Tuberculosis, and Malaria totaled approximately 4 million (5). A recent Lancet commission has shed light on the importance of global surgery, emphasizing an evidencebased approach for the development of surgical capacity in low and middle-income countries (LMIC) (6).

Unfortunately, there is limited data on the prevalence of global urologic disease. We do know, however, that a significant portion of undertreated global disease is urologic - urinary retention, urethral stricture, malignancy, and urolithiasis (7). Much of the effort to improve urologic care worldwide has come in the form of international volunteerism. The phenomenon of medical volunteerism is deeply rooted in the altruistic ideals and culture of medicine and surgery. In recent years, however, it has become more evident that development and relief efforts worldwide must be accomplished in specific ways to engender tangible and sustainable change for the better. In order to improve urologic care globally, there must be a concerted effort to first understand and define the burden of specific urologic 
conditions and secondarily to mobilize collaborative, systematic efforts for change. With this in mind, we aim to review the burden of disease globally, its main etiologies, and important considerations for care in resource-poor environments. We focus on urethral stricture as it is a common urologic problem in the global health arena that, if treated appropriately, can be managed with durable results.

\section{State of urologic care worldwide}

Globally, access to and quality of urologic care varies greatly from region to region. For instance, the 2016 American Urological Association (AUA) census reported that the overall urologist to population ratio in the United States was 3.77 per 100,000 persons (8). In stark contrast, IVUmed, an organization that sends urologic volunteers all over the world, reports that there are less than 30 urologists for the entire population of Kenya, a country of greater than 41 million individuals (9). Additionally, the British Association of Urological Surgeons (BAUS) estimates that there is one urologist per 2.3 million people in Zambia while in the UK they estimate one urologist per 72,189 individuals (7). With such steep disparities in healthcare workforce, it is no surprise that there is a substantial unmet need in middle and lower-income countries. Explanations for the lack of healthcare workforce in developing countries are multifaceted. Contributing factors include lack of appropriate and necessary healthcare education, local and international migration of skilled workers, lack of underlying infrastructure to support training and practice of healthcare workers, and lack of baseline data leading to difficulty in policy development (10).

There have been many proposed strategies for building workforce capacity in low-income countries around the globe. Many of the proposed approaches involve changes in the culture and infrastructure of healthcare organizations in developing countries. Focusing on the fundamental infrastructural landscape of healthcare systems will lead to stable training and operational environment for local workers. International volunteerism has been used to supplement this development and to provide relief in times of growth.

\section{Global burden of urologic and urethral stricture disease}

Data assessing the burden of urethral stricture and reconstructive urologic disease worldwide is scarce. The data that does exist is unfortunately incomplete by its very nature. Many countries do not have the infrastructure to maintain this kind of data, and patients around the world have varying access to medical care making underreporting of disease prevalence a global issue. More broadly, it is clear that surgical disease represents a large fraction of overall disease burden. Surgical conditions account for up to $30 \%$ of the total global burden of disease. Furthermore, as reported by Weiser et al. in 2008, approximately 230 million surgeries are performed worldwide in a given year (11). As the authors point out, this exceeds the annual volume of childbirth by almost double. Rose et al. calculated the estimated worldwide need for surgical cases based on available census and prevalence data which totaled approximately 320 million (12). This leaves a large unmet need (calculated at approximately 140 million cases in the Lancet commission publication) that includes a large fraction of urologic burden (6). Further characterization at this time is difficult due to paucity of data.

Despite this lack of data, it is clear that genitourinary pathology is a significant contributor to worldwide medical and surgical disease burden. Irwin et al. estimated the worldwide prevalence of lower urinary tract symptoms at $45.2 \%$ in 2008 (13). According to Campain et al., a survey of 48 urologists working in sub-Saharan Africa ranked the top most commonly encountered urologic conditions as $\mathrm{BPH}$, urethral stricture, prostate cancer, bladder cancer, and urethral/ureteral trauma (7). Attempts have been made to quantify the prevalence of urethral stricture disease specifically in certain areas of the world. Santucci et al. queried data from ten public and private databases in the United States looking for the incidence of urethral stricture disease. They estimated an incidence of $0.6 \%$ in those susceptible to the disease (14). Further analysis from the same group revealed a drop in incidence of urethral stricture from $1.4-0.9 \%$ in the Medicare population from 1992-2001 (15). These may have been secondary to improvement in urologic techniques which cause iatrogenic stricture. The international consultation on Urethral Stricture held in 2010 stated there are no currently available direct measures of the worldwide incidence of urethral stricture (16). The current lack of epidemiologic data worldwide leaves us to extrapolate from current data, placing the incidence of urethral stricture between $0.6 \%$ and $1.4 \%$.

\section{Global management of urethral stricture}

Given the estimated burden of disease, strategies must 
Table 1 Urethral stricture etiology: HIC vs. LMIC

LMIC
Lrauma
Infectious
Idiopathic
latrogenic
HIC
Idiopathic
latrogenic
Trauma
Lichen sclerosis
Infectious
Difference in etiology of urethral stricture in high income
countries (HIC) versus low and middle income countries (LMIC).
Listed from most common to least common etiology based on
review of current literature (17-21).

be developed to tackle the global problem of urethral stricture systematically. Much that we have learned in the management of urethral stricture through research in highincome settings may be applied to LMICs. Previously developed treatment algorithms, procedural techniques, and therapeutic philosophy may be relied upon for guidance. Given the differences in etiology, healthcare infrastructure, and healthcare worker capacity in the global population there must be an emphasis on approaches that fit with local community complexity. Here we review the etiology and management of urethral stricture with specific strategies for the global community.

The natural history of urethral stricture has been well elucidated. When left untreated, urethral stricture can lead to significant morbidity including trabeculated bladder, urinary retention, hydronephrosis, urethral abscess, and urinary tract calculi (14). Effective and early correction of urethral stricture leads to years of improved urinary tract function and quality of life. The management of urethral stricture is fundamentally surgical. Aside from providing symptomatic relief, medical therapies are not useful in treating urethral strictures. Stricture disease, therefore, represents an opportunity for large-scale development of surgical based quality improvement in LMICs.

Etiology of urethral stricture varies by geographical region. Stein et al. reviewed data from the United States,
Italy, and India to understand the difference in etiology of urethral stricture by geographic region. Group 1 was comprised of 1,900 men from Italy and the United States while 715 men from India formed group 2. The groups differed significantly concerning etiology of urethral stricture. Group 1 had a higher incidence of penile strictures secondary to iatrogenic causes whereas group 2 had significantly greater traumatic strictures and strictures secondary to lichen sclerosis (17). Various epidemiologic studies confirm these findings. Fenton et al. retrospectively reviewed 150 patients who underwent anterior urethral stricture repair at a medical center in the United States. They found that the etiology of the majority of strictures were idiopathic or iatrogenic (18). Palminteri et al. reviewed 1,439 patients in Italy with urethral stricture and also found that the most common etiologies were iatrogenic and idiopathic (19). A recent retrospective review from Nigeria looked at 46 patients treated for urethral stricture between 2006 and 2016. They found that majority of strictures were secondary to infectious and iatrogenic etiology (20). Tijani et al. looked at the etiology of urethral stricture disease in 42 patients between 1998 and 2005 in Lagos, Nigeria. In that study, the most common etiology of stricture was accidental urethral trauma (21). These differences in etiology highlight the need to tailor urologic care for and prevention of stricture disease to the particular region in questions (Table 1).

Options for treatment of urethral stricture vary by location and etiology. Generally, however, the most common management strategies for all types of stricture include endoscopic techniques (dilation, incision, and injection) and open surgical repair via urethroplasty. It has been well established that urethroplasty for the treatment of urethral stricture offers the highest and most durable success rate (22). Urethroplasty technique varies widely depending on the location and severity of the stricture. Faris et al. retrospectively assessed the success rates of urethroplasty for urethral stricture and determined that the caseload necessary to achieve high success rates $(>90 \%)$ was about 100 cases (23). Fossati et al. assessed the success rate for anterior one-stage urethroplasty and found a statistically significant increase in success with surgeon experience (24). These findings are especially important in LMICs where the fellowship-trained reconstructive urologist is rare. Though repeat urethroplasty is possible with a high success rate in experienced hands, success rates decline with each repeat surgery making the first urethroplasty the best chance for the patient (25).

Urethral stricture represents a unique case study in 
actionable global surgical disease given its progressive natural history and its surgical therapeutic management. As detailed previously, geographic variation in etiology of urethral stricture and the paucity of trained reconstructive urologists around the globe are important considerations. Many studies have demonstrated that surgical specialization and increased surgical volume leads to better results (26-28). This has led to increasing surgical sub-specialization in high-income countries such as the United States. Similar principles can be applied to LMICs. This model may be tailored to the local region, where tertiary centers are established as referral centers for community workers. Centers like these have already been established in countries such as Senegal and Haiti by varying organizations, including IVUmed (29). The hope is that these centers will allow for the greatest benefit per patient with urethral stricture.

The above-proposed model relies on a wide infrastructural network of referring providers. The AUA guideline on male urethral stricture disease recommends diagnosis and treatment based on modalities that may not be available in lowincome settings such as urethrography and cystoscopy (30). Patients may be referred to tertiary centers for both testing and treatment, but community-based providers must then be educated on the signs and symptoms of urethral stricture as well as appropriate bladder decompression techniques, including suprapubic cystostomy, to be used in patients presenting with urinary retention. Many LMICs lack this infrastructure, and many community health workers may not be comfortable with these procedural techniques. This represents an opportunity for intervention. Collaborative international volunteerism focusing on education and sustainable development offers a key solution in providing LMICs with the capacity to improve care for urethral stricture disease on a global scale.

Another strategy to increase fellowship trained reconstructive urologists in LMICs, is to train foreign medical graduates with the hope of them returning to practice in their home country. This may be more appealing as job saturation for reconstructive urology in the highincome country (HIC) becomes increasingly problematic.

\section{International volunteerism}

Many solutions have been proposed to address the needs of LMICs. One of the most popular solutions in recent history has been international volunteerism, whereby providers travel to resource-poor areas in an effort to provide medical assistance. These types of interventions have grown from individual efforts of single practitioners to institutional partnerships hoping to provide sustainable, multifaceted assistance. There is a wide range of literature discussing the various efforts by groups in the surgical world participating in these types of efforts (31-33). Our review found less literature in the urologic world. While published literature is scarce, multiple organizations are championing the cause of global urologic care. The most widely recognized organizations include IVUmed, Urolink, The Global Philanthropic Committee (GPC) (Table 2).

IVUmed seeks to provide surgical education in resourcepoor environments focusing on urologic procedures (Table 3). It was established By Dr. Catherine DeVries in 1995 after participating in international education and development. IVUmed's stated goal is to provide global education in urologic surgery and has developed relationships with urologic programs worldwide, sending teams of urologists from different subspecialties to educate local surgeons on the most up to date techniques and management strategies (Figure 1). Urolink is an organization that is closely affiliated with the BAUS. They have been active in the global surgery arena since 1990 . Their mission is to "To promote and encourage the provision of appropriate urological expertise and education worldwide with particular emphasis on the materially disadvantaged." (34). Their mission has been divided further into five areas of activity including encouraging the development of links between entities, facilitating professional visits to areas of need, supporting urologic training, assisting with provision of equipment, and advising the BAUS council on matters involving the developing world. The GPC is a collaboration between urologic organizations including the American Urological Association (AUA), the European Urological Association (EUA), the International Continence Society (ICS), and the Société Internationale d'Urologie (SIU). The GPC seeks to fund global urologic philanthropic projects that provide large-scale capacity for change. The development of organized international volunteer efforts will only continue to improve the individual labors of medical professionals around the globe.

\section{Ethical considerations}

While altruism often motivates international volunteer efforts, ethical considerations must be taken into account when embarking on these endeavors. It has been estimated that there are over 500 organizations that partake in medical 
Table 2 Summary of major international volunteer organizations in urology

\begin{tabular}{|c|c|c|c|}
\hline Organization & Year established & Stated mission & Brief description \\
\hline IVUmed & 1990 & $\begin{array}{l}\text { IVUmed is committed to making quality } \\
\text { urological care available to people worldwide. In } \\
\text { fulfilling this mission, IVUmed provides medical } \\
\text { and surgical education to physicians and nurses } \\
\text { and treatment to thousands of children and } \\
\text { adults }\end{array}$ & $\begin{array}{l}\text { Focuses on sustainable training of surgical } \\
\text { process and urologic procedures using a } 5 \text {-year } \\
\text { structures workshop approach in which volunteer } \\
\text { experts train local surgeons who eventually gain } \\
\text { the expertise needed to care for complex cases }\end{array}$ \\
\hline Urolink & 1995 & $\begin{array}{l}\text { To promote and encourage the provision of } \\
\text { appropriate urological expertise and education } \\
\text { worldwide with particular emphasis on the } \\
\text { materially disadvantaged }\end{array}$ & $\begin{array}{l}\text { Focus on } 5 \text { key areas of activity including } \\
\text { establishing links between entities, encouraging } \\
\text { overseas visits, supporting urological training, } \\
\text { assisting with provision of equipment, and } \\
\text { advising the BAUS council on matters relating to } \\
\text { the developing world }\end{array}$ \\
\hline
\end{tabular}

AUA, American Urological Association; EAU, European Association of Urology; ICS, International Continence Society; BAUS, British Association of Urological Surgeons.

Table 3 IVUmed global impact statistics for 2016

\begin{tabular}{ll}
\hline Metric & Total \\
\hline Medical professionals trained & 401 \\
Surgical workshops & 22 \\
Partner countries & 14 \\
Donated volunteer hours & 11,153 \\
Patients served & 727 \\
\hline
\end{tabular}

volunteerism to some extent, representing approximately 250 million dollars of expenditure per year (35). International volunteer efforts, especially short-term medical service trips, have come under increasing scrutiny due to the genuine possibility of doing more harm than good. The dynamic between the volunteer and the community can be one of mutual benefit if done thoughtfully. Much too often, however, the intended benefit to the community is unrealized or more harm is done than good. Bauer recently reviewed the ethical dilemmas engrained in the history of international volunteerism, including doing real harm, ethical double standards, and accounts of volunteer efforts gone awry (36). For example, international volunteer efforts may fail to identify the priorities of the local community, which may end up leaving locals with unnecessary and unrealistic end products. Furthermore, efforts that fail to blend with the local economic environment may lead to loss of local jobs and dependence on foreign aid. Additionally, lack of understanding of cultural beliefs and norms may fray the fabric of the local culture and lead to a watering down of local identity. Ethical considerations also arise when sending trainees to low resource environments, implying responsibility for oversight that may not be met. Although great potential exists for development and progress by way of international volunteerism and international development, ethical principles must be actively considered during the engineering of such efforts.

\section{Conclusions}

The recent Lancet commission publication highlights the critical steps necessary to improve global surgical care. The understanding of global urethral stricture disease is limited due to a lack of data. Current understanding, however, highlights the fact that stricture disease abroad is more commonly due to trauma in LMICs than in developed countries. Furthermore, knowing that urethral strictures do better when correctly reconstructed the first time, we can devise practice algorithms that utilize the limited capacity efficiently by developing tertiary referral centers that have been trained in reconstructive urology. Through this paradigm, it may be possible to reach as many individuals as possible, even in the most resource-poor environments. Ethical considerations are paramount when embarking on 


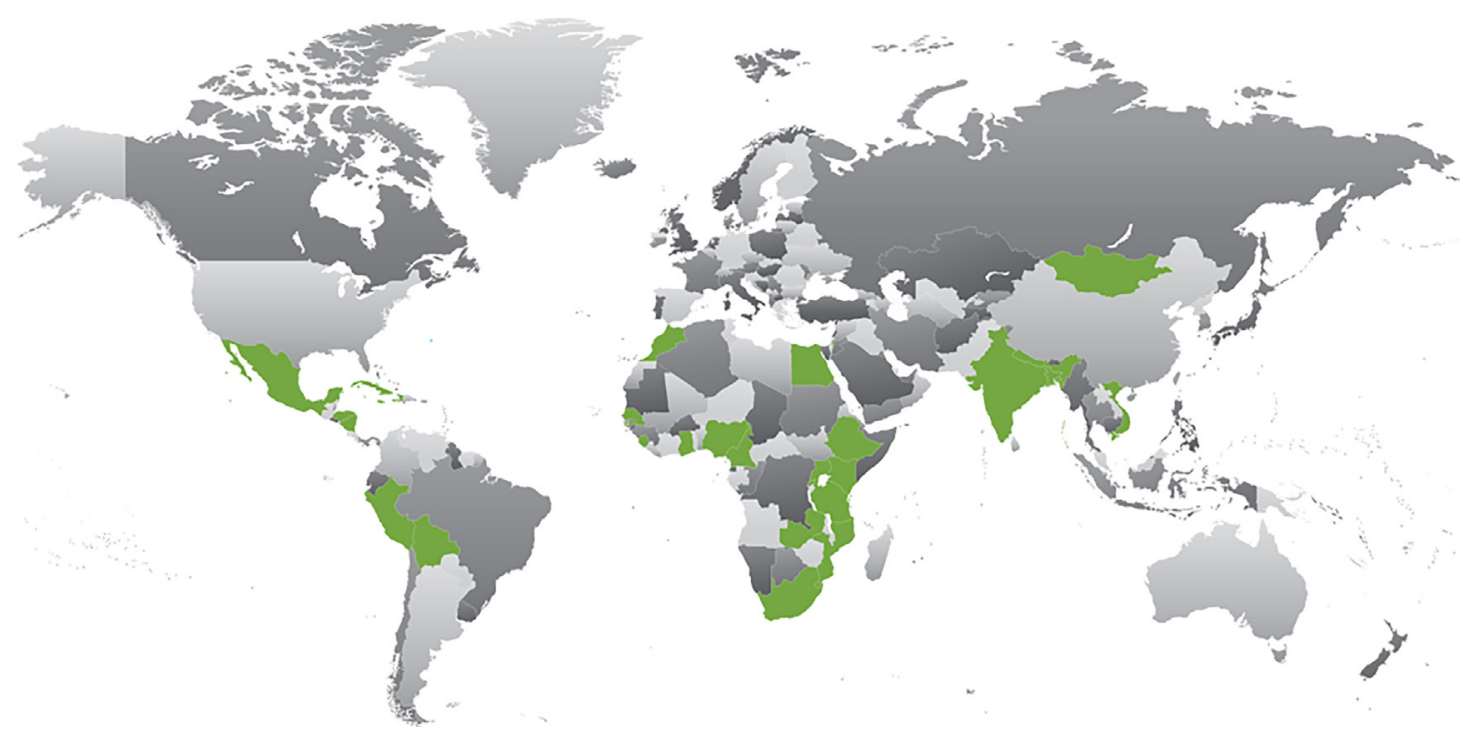

Figure 1 Map depicting countries in which IVUmed operates. Printed with IVUmed permission.

international development efforts. Further concentration on infrastructure, capacity building, and collaboration between international volunteers are necessary to improve care for those afflicted by urethral stricture worldwide.

\section{Acknowledgements}

None.

\section{Footnote}

Conflicts of Interest: The authors have no conflicts of interest to declare.

\section{References}

1. General AUN. United Nations Millennium Declaration, Resolution Adopted by the General Assembly. UN General Assembly. 2000. Available online: http://www. un.org/millennium/declaration/ares552e.pdf, accessed 28 Nov 2017 A/RES/55/2.

2. Molyneux DH, Hotez PJ, Fenwick A. "Rapid-impact interventions": how a policy of integrated control for Africa's neglected tropical diseases could benefit the poor. PLoS Med 2005;2:e336.

3. Nations U. The Millennium Development Goals Report. New York, 2015.

4. Shrime MG, Bickler SW, Alkire BC, et al. Global burden of surgical disease: an estimation from the provider perspective. Lancet Glob Health 2015;3 Suppl 2:S8-9.

5. Lozano R, Naghavi M, Foreman K, et al. Global and regional mortality from 235 causes of death for 20 age groups in 1990 and 2010: a systematic analysis for the Global Burden of Disease Study 2010. Lancet 2012;380:2095-128.

6. Meara JG, Leather AJ, Hagander L, et al. Global Surgery 2030: evidence and solutions for achieving health, welfare, and economic development. Lancet 2015;386:569-624.

7. Campain NJ, MacDonagh RP, Mteta KA, et al. Global surgery - how much of the burden is urological? BJU Int 2015;116:314-6.

8. American Urological Association. The State of Urology Workforce and Practice in the United States 2016. In: AUA Census. American Urological Association. 2017. Available online: https://www.AUAnet.org/common/pdf/ research/census/State-Urology-Workforce-Practice-US. pdf, accessed 11/22/17.

9. IVUmed. Kenya. 2017. Available online: https://www.ivumed. org/where-we-work/kenya/, accessed 11/22/17 2017.

10. Guilbert JJ. The World Health Report 2006: working together for health. Educ Health (Abingdon) 2006;19:385-7.

11. Weiser TG, Regenbogen SE, Thompson KD, et al. An estimation of the global volume of surgery: a modelling strategy based on available data. Lancet 2008;372:139-44.

12. Rose J, Weiser TG, Hider P, et al. Estimated need for surgery worldwide based on prevalence of diseases: a modelling strategy for the WHO Global Health Estimate. 
Lancet Glob Health 2015;3 Suppl 2:S13-20.

13. Irwin DE, Kopp ZS, Agatep B, et al. Worldwide prevalence estimates of lower urinary tract symptoms, overactive bladder, urinary incontinence and bladder outlet obstruction. BJU Int 2011;108:1132-8.

14. Santucci RA, Joyce GF, Wise M. Male urethral stricture disease. J Urol 2007;177:1667-74.

15. Anger JT, Santucci R, Grossberg AL, et al. The morbidity of urethral stricture disease among male medicare beneficiaries. BMC Urol 2010;10:3.

16. Jordan G, Chapple C, Heyns C. An International Consultation on Urethral Strictures. October 13-16, 2010; Marrakech, Morocco. Available online: https://www.siuurology.org/themes/web/assets/files/ICUD/pdf/urethral_ strictures_2010.pdf

17. Stein DM, Thum DJ, Barbagli G, et al. A geographic analysis of male urethral stricture aetiology and location. BJU Int 2013;112:830-4.

18. Fenton AS, Morey AF, Aviles R, et al. Anterior urethral strictures: etiology and characteristics. Urology 2005;65:1055-8.

19. Palminteri E, Berdondini E, Verze P, et al. Contemporary urethral stricture characteristics in the developed world. Urology 2013;81:191-6.

20. Irekpita E. A 10-Year Review of Urethral Stricture Management in Irrua, Nigeria. Niger J Surg 2017;23:119-24.

21. Tijani KH, Adesanya AA, Ogo CN. The new pattern of urethral stricture disease in Lagos, Nigeria. Niger Postgrad Med J 2009;16:162-5.

22. Wong SS, Narahari R, O'Riordan A, et al. Simple urethral dilatation, endoscopic urethrotomy, and urethroplasty for urethral stricture disease in adult men. Cochrane Database Syst Rev 2010;(4):CD006934.

23. Faris SF, Myers JB, Voelzke BB, et al. Assessment of the Male Urethral Reconstruction Learning Curve. Urology 2016;89:137-42.

Cite this article as: Frankel JK, Murphy GP. International volunteerism and urethral stricture disease: a review. Transl Androl Urol 2018;7(4):659-665. doi: 10.21037/tau.2018.02.04
24. Fossati N, Barbagli G, Larcher A, et al. The Surgical Learning Curve for One-stage Anterior Urethroplasty: A Prospective Single-surgeon Study. Eur Urol 2016;69:686-90.

25. Blaschko SD, McAninch JW, Myers JB, et al. Repeat urethroplasty after failed urethral reconstruction: outcome analysis of 130 patients. J Urol 2012;188:2260-4.

26. Birkmeyer JD, Stukel TA, Siewers AE, et al. Surgeon volume and operative mortality in the United States. N Engl J Med 2003;349:2117-27.

27. Reames BN, Ghaferi AA, Birkmeyer JD, et al. Hospital volume and operative mortality in the modern era. Ann Surg 2014;260:244-51.

28. Sahni NR, Dalton M, Cutler DM, et al. Surgeon specialization and operative mortality in United States: retrospective analysis. BMJ 2016;354:i3571.

29. Badlani G. International volunteerism and global responsibility. Transl Androl Urol 2017;6:258-63.

30. Wessells H, Angermeier KW, Elliott S, et al. Male Urethral Stricture: American Urological Association Guideline. J Urol 2017;197:182-90.

31. Shrime MG, Sleemi A, Ravilla TD. Charitable platforms in global surgery: a systematic review of their effectiveness, cost-effectiveness, sustainability, and role training. World J Surg 2015;39:10-20.

32. Casey KM. The global impact of surgical volunteerism. Surg Clin North Am 2007;87:949-60, ix.

33. Wolfberg AJ. Volunteering overseas--lessons from surgical brigades. N Engl J Med 2006;354:443-5.

34. Harrison NW. UROLINK: a model for working together in a changing world. BJU Int 2002;89 Suppl 1:1-5.

35. Maki J, Qualls M, White B, et al. Health impact assessment and short-term medical missions: a methods study to evaluate quality of care. BMC Health Serv Res 2008;8:121.

36. Bauer I. More harm than good? The questionable ethics of medical volunteering and international student placements. Trop Dis Travel Med Vaccines 2017;3:5. 